\title{
Aspectos socioeconômicos no contexto de doenças tropicais negligenciadas nos municípios de Abaetetuba e Barcarena, Pará
}

As Doenças Tropicais Negligenciadas (DTNs) constituem um grupo de condições que causam morbidade, incapacidade crônica e morte, tendo como população mais afetada aquela que vive em vulnerabilidade socioeconômica, por carecer de serviços básicos de água, higiene e saneamento básico. O objetivo deste estudo foi analisar o contexto das doenças tropicais negligenciadas sob os aspectos socioeconômicos no município de Abaetetuba e Barcarena, Pará. A metodologia utilizada foi estudo ecológico, descritivo, de série temporal, no período entre 2007 e 2016, cujas variáveis analisadas foram a incidência das doenças notificadas, segundo as Secretarias Municipais de Saúde de ambos os municípios, e o Índice de Desenvolvimento Humano Municipal (IDHM). Foram notificados mais de 7 mil casos de DTNs em Abaetetuba e mais de 1700 casos em Barcarena, sendo elas leishmaniose tegumentar americana, leishmaniose visceral, doença de Chagas, tuberculose, hanseníase e dengue, sendo que os dados relacionados à dengue foram disponibilizados apenas pela Secretaria Municipal de Saúde de Barcarena. 0 município de de Chagas, tuberculose, hanseniase e dengue, sendo que os dados relacionados à dengue foram disponibilizados apenas pela Secretaria Municipal de Saúde de Barcarena. O municipio de Abaetetuba apresentou os maiores índices de incidência das DTNs, principalmente para doença de Chagas, leishmaniose visceral e hanseníase; apresentou também um número populacional maior que Barcarena, embora este tenha tido um crescimento populacional bem maior que Abaetetuba no período entre 2007 e 2016. Em Barcarena, as DTNs com maiores incidências são

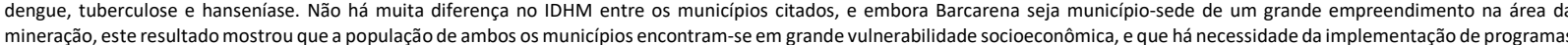
que visem a erradicação destes agravos, assim como ampliação dos investimentos em educação e saneamento básico para que as iniquidades sociais sejam minimizadas.

Palavras-chave: Doenças Negligenciadas; Índices Socioeconômicos; Vulnerabilidade.

\section{Socioeconomic aspects in the context of neglected tropical diseases in the municipalities of Abaetetuba and Barcarena, Pará}

\begin{abstract}
Neglected Tropical Diseases (NTDs) are a group of conditions that causes chronic disability and death, with the population most affected by those who live in socioeconomic vulnerability considering basic water, hygiene and basic sanitation services. The objective of this study was to analyze the context of neglected climatic diseases under socioeconomic aspects in the municipality of Abaetetuba and Barcarena, Pará. The methodology used was the ecological, descriptive, time series study, between 2007 and 2016, and the variables Analyzed were the incidence of notified diseases, according to the Secretaries of Municipal Health Secretariats and the Municipal Human Development Index (MHDI). More than 7 thousand cases of NTDs have incidence of notified diseases, according to the Secretaries of Municipal Health Secretariats and the Municipal Human Development Index (MHDI). More than 7 thousand cases of NTDs have
been reported in Abaetetuba and more than 1700 cases in Barcarena, namely American cutaneous leishmaniasis, visceral leishmaniasis, Chagas disease, tuberculosis, leprosy and dengue, with data related to dengue only being made available by the Secretary of Municipal Health Council in Barcarena. The municipality of Abaetetuba had the highest incidence rates of NTDs, mainly for Chagas disease, visceral leishmaniasis and leprosy; it also had a larger population number than Barcarena, although it had a much larger population growth than Abaetetuba in the period between 2007 and 2016. In Barcarena, as NTDs with the highest incidence are dengue, tuberculosis and leprosy. There is not much difference in the MHDI between the mentioned municipalities, and although Barcarena is the host city of a large mining enterprise, this result showed that the population of both municipalities are in great socioeconomic vulnerability, and that there is a need for implementation of programs aimed at eradicating these diseases, as well as expanding investments in education and basic sanitation so that social inequities are minimized.
\end{abstract}

Keywords: Neglected Diseases; Socioeconomic Indexes; Vulnerability.

Topic: Epidemiologia e Saúde Ambiental

Reviewed anonymously in the process of blind peer.
Received: 02/08/2020

Approved: 19/09/2020
Alcione Pinheiro de Oliveira (D)

Universidade do Estado do Pará, Brasil

http://lattes.cnpq.br/7480135822996536

http://orcid.org/0000-0002-0587-4204

oliveiraalcy05@gmail.com

Thamires Beatriz dos Santos Caitano (iD)

Universidade do Estado do Pará, Brasil

http://lattes.cnpq.br/4649556839610299

http://orcid.org/0000-0002-7538-4366

thamirescaitano@hotmail.com

Altem Nascimento Pontes (iD)

Universidade do Estado do Pará, Brasil

http://lattes.cnpq.br/5993352890364998

http://orcid.org/0000-0002-9001-4603

altempontes@gmail.com
Referencing this:

OLIVEIRA, A. P.; CAITANO, T. B. S.; PONTES, A. N.. Aspectos socioeconômicos no contexto de doenças tropicais negligenciadas nos municípios de Abaetetuba e Barcarena, Pará. Revista Ibero Americana de Ciências Ambientais, v.11, n.5, p.237-248, 2020. DOI: http://doi.org/10.6008/CBPC2179-6858.2020.005.0023 


\section{INTRODUÇÃO}

As Doenças Tropicais Negligenciadas (DTNs) constituem um grupo de condições que causam morbidade, incapacidade crônica e morte, além de estarem relacionadas a impactos socioeconômicos (ENGELMAN et al., 2016). As populações mais afetadas são as mais pobres, que carecem dos serviços mais básicos de água, higiene e saneamento básico, com notável aglomeração geográfica, prejudicando, assim, a vida de mais de um bilhão de pessoas (HOFSTRAAT, 2016).

De acordo com a Organização Mundial da Saúde (WHO, 2015), o grupo de doenças tropicais negligenciadas compreende cerca de 20 patologias, como tuberculose, doença de Chagas, leishmanioses, dengue, além de envenenamento por picadas de cobras. Estima-se que cerca de 80 milhões de pessoas vivam em áreas onde uma ou mais DTNs sejam endêmicas (WHO, 2017).

Existem cinco intervenções de saúde pública para controle e erradicação das doenças negligenciadas, tendo destaque o controle de vetores, água potável e saneamento básico (WHO, 2017). No entanto, alguns fatores como as alterações socioambientais, a migração populacional sazonal e a ausência de políticas efetivas orientadas ao enfrentamento destas doenças influenciam de modo negativo nestas intervenções (BUCKEE et al., 2017; BOOTH et al., 2018; SOBRAL et al., 2018).

Segundo Mengitsua et al. (2016), países como a Etiópia lideraram o aumento de intervenções para lidar com grande parte dessa população em risco e atingir as metas de eliminação e controle de DTNs até 2020. Há programas para a erradicação de outras patologias negligenciadas em outros países, como Sudão do Sul e Síria (JACOBSON et al., 2018). Na América Latina, o Brasil é o país com maior número de casos e maior carga de doenças relacionadas às doenças negligenciadas (BRASIL, 2018a). Como medida de combate foram definidas sete prioridades de atuação que compõem o Programa de Pesquisa e Desenvolvimento em Doenças Negligenciadas, dentre as quais recebem destaque dengue, doença de Chagas, hanseníase, leishmanioses e tuberculose (INÁCIO et al., 2019).

O Ministério da Saúde afirma que o Brasil apresentou redução das prevalências das doenças tropicais, desde 2011, quando houve intensificação das ações de prevenção e controle das seis DTNs que compõem o Plano Integrado de Ações Estratégicas (BRASIL, 2012). Entretanto, na região amazônica foram registrados novos casos de leishmaniose tegumentar americana, doença de Chagas e dengue/chikungunya/zika, sendo o Amazonas, o Pará e o Tocantins os estados com maiores números de notificações, respectivamente (TELES et al., 2019).

Com isso, percebe-se que a Amazônia faz parte de um cenário negligenciado pelo poder público, sendo vista apenas como local para implementação de indústrias, extraindo a matéria-prima necessária e causando impactos socioambientais, que acabam prejudicando a interação homem-natureza, além de colaborar com o surgimento de doenças e piorar a situação de pobreza nesta região (MEDEIROS et al., 2016; SOUZA, 2018). Pautado em um discurso de desenvolvimento e modernização, a exploração nem sempre está condizente com o bem-estar social (ARAÚJO et al., 2009; BRANDÃO et al., 2018).

A exemplo disso, tem-se a criação da empresa Alumínio Brasileiro S. A. (Albrás), em 1985, que 
contribuiu para que o crescimento populacional tanto no município de Barcarena quanto nos municípios vizinhos, como Abaetetuba, fosse bastante expressivo, recebendo migrantes, em sua maioria, nordestinos, que, em busca de emprego, contribuíram de forma abundante com a mão-de-obra barata para as atividades de baixa qualificação e insalubres produzidas pelo projeto (ALVES et al., 2015; QUARESMA et al., 2015).

A implementação da indústria no contexto amazônico contribuiu com o rápido crescimento populacional, a urbanização e a perda de biodiversidade, acarretando a construção de novas periferias sem estrutura básica (BRANDÃO et al., 2018). O número de pessoas que adoecem nessas áreas constitui uma relação direta entre a prevalência de DTNs e o baixo Índice de Desenvolvimento Humano Municipal (IDHM) (BOOTH et al., 2018; BRASIL, 2018a). O objetivo deste estudo foi analisar o contexto das doenças tropicais negligenciadas sob os aspectos socioeconômicos nos municípios de Abaetetuba e Barcarena, Pará, para que assim seja possível a elaboração de políticas públicas viáveis e eficazes para o controle e/ou erradicação destes agravos.

\section{METODOLOGIA}

\section{Área de estudo}

Este estudo foi realizado nos municípios de Abaetetuba e Barcarena (Figura 1), ambos localizados no Nordeste paraense, com população estimada, em aproximadamente, 157 e 124 mil habitantes, cada, e apresentando uma densidade demográfica de cerca de 97 e 95 hab $/ \mathrm{km}^{2}$, respectivamente (IBGE, 2019).

O município de Abaetetuba situa-se à margem direita do rio Maratauíra, pertencendo à microrregião de Cametá e à mesorregião do Nordeste paraense. As coordenadas geográficas da sede indicam $01^{\circ} 43^{\prime} 31^{\prime \prime}$ de latitude Sul e $48^{\circ} 53^{\prime} 31^{\prime \prime}$ de longitude a Oeste de Greenwich, tendo como limite ao Norte os municípios de Barcarena; a Leste, Moju; ao Sul, Igarapé-Miri e a Oeste, Moju, Igarapé-Miri, Limoeiro do Ajuru e Muaná, conforme observado na Figura 1 (ABAETETUBA, 2017).

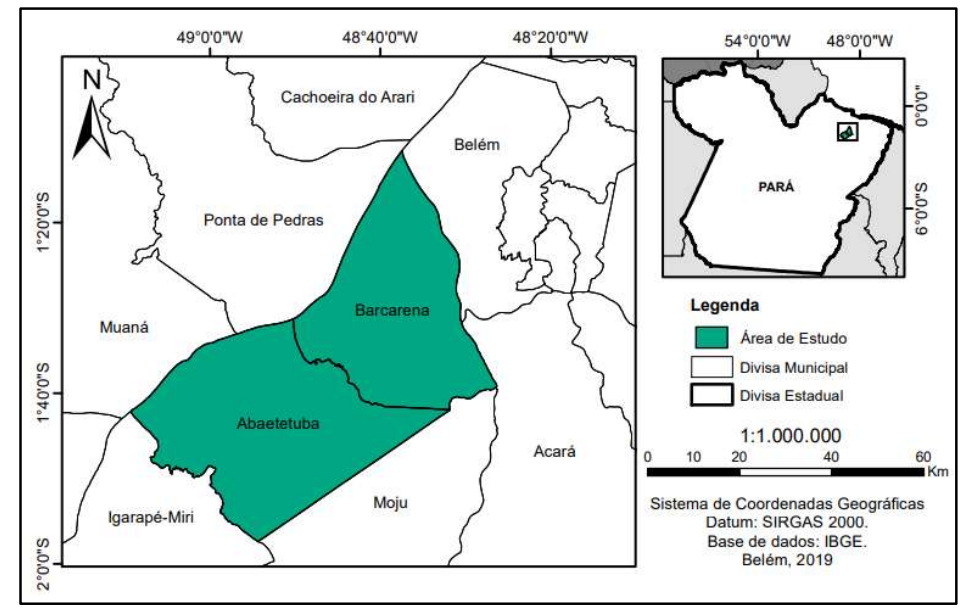

Figura 1: Mapa dos municípios de Abaetetuba e Barcarena, Pará, Brasil.

O município de Barcarena pertence à mesorregião metropolitana de Belém e à microrregião de Belém, apresentando as seguintes coordenadas geográficas: $01^{\circ} 30^{\prime} 24^{\prime \prime}$ de latitude Sul e $48^{\circ} 37^{\prime} 12^{\prime \prime}$ de longitude a Oeste de Greenwich. Os limites deste município compreendem ao Norte, a baía de Guajará e 
município de Belém; ao Sul, os municípios de Moju e Abaetetuba; ao Leste, a baía de Guajará e município de Acará, e a Oeste, a baía do Marajó (FAPESPA, 2017).

\section{Coleta e análise de dados}

Foi realizado um estudo ecológico, descritivo, de série temporal, no período entre 2007 e 2016 acerca das doenças tropicais negligenciadas nos municípios de Abaetetuba e Barcarena, no estado do Pará. As variáveis estudadas foram a incidência das doenças notificadas e o Índice de Desenvolvimento Humano Municipal (IDHM). Estes dados foram obtidos, respectivamente, via Secretarias Municipais de Saúde e Índice FIRJAN de Desenvolvimento Municipal (IFDM) (FIRJAN, 2017). O IFDM foi criado devido à necessidade de se monitorar o desenvolvimento socioeconômico de uma região, avaliando, assim, as três principais áreas de desenvolvimento humano: Emprego e Renda, Educação e Saúde. A leitura dos resultados varia de 0 a 1 , de modo que quanto mais próximo de 1, maior será o nível de desenvolvimento da localidade (FIRJAN, 2017).

As informações ligadas ao quantitativo populacional e demográfico foram obtidas por meio do Instituto Brasileiro de Geografia e Estatística (IBGE) e da Fundação Amazônia de Amparo a Estudos e Pesquisas (FAPESPA). A FAPESPA elaborou documentos intitulados Estatísticas Municipais Paraenses, onde são traçados os perfis municipais com seus aspectos socioeconômicos e estatísticos, por entender que com a organização e disponibilidade destes dados, aumenta-se a possibilidade de acertos na tomada de decisões (FAPESPA, 2017).

Os dados foram organizados em planilhas Excel 2013 e depois foi empregada a estatística descritiva para elaboração de tabelas e figuras. Foram calculadas taxas de incidência e seu coeficiente levando em consideração cada agravo notificado e a população estimada, entre 2007 e 2016, além de que, entre 2007 e 2010, a população do município de Barcarena não atingia 100.000 habitantes, sendo utilizado o valor de 10.000 habitantes neste cálculo.

\section{RESULTADOS}

\section{Doenças tropicais negligenciadas notificadas}

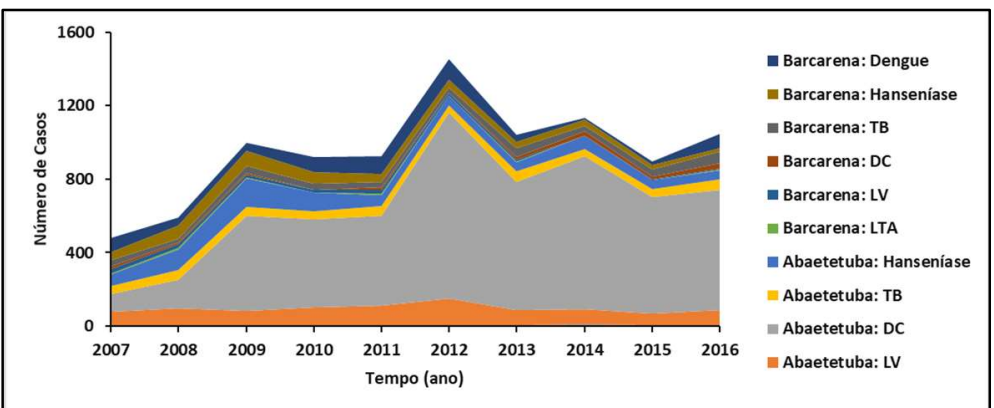

Figura 2: Doenças Tropicais Negligenciadas notificadas nos municípios de Abaetetuba e Barcarena, Pará.

De acordo com os dados fornecidos pelas Secretarias Municipais de Saúde de Abaetetuba e Barcarena, entre 2007 e 2016 foram notificados mais de 7.000 casos de doenças negligenciadas em Abaetetuba e mais de 1.700 casos em Barcarena. As doenças notificadas foram leishmaniose tegumentar 
americana (LTA), leishmaniose visceral (LV), doença de Chagas (DC), tuberculose (TB), hanseníase e dengue, sendo esta última apenas para o município de Barcarena, conforme mostra a Figura 2.

Os agravos que tiveram maiores notificações obtiveram também as maiores taxas de incidência (doença de Chagas, leishmaniose visceral, hanseníase, dengue e tuberculose). As Tabelas 1 e 2 mostram a taxa de incidência de cada agravo notificado por ano, em cada um dos municípios.

Tabela 1: Taxa de incidência de cada agravo notificado por ano, Abaetetuba, Pará, Brasil.

\begin{tabular}{llllll}
\hline \multirow{2}{*}{ ANO } & \multicolumn{6}{l}{ DOENÇAS TROPICAIS NEGLIGENCIADAS } & \multicolumn{3}{l}{} \\
\cline { 2 - 6 } & LTA & LV & DC & TB & HANSENÍASE \\
\hline 2007 & 0,30 & 5,47 & 7,20 & 3,37 & 4,87 \\
2008 & 0,14 & 6,81 & 11,37 & 3,84 & 8,18 \\
2009 & 0,07 & 5,93 & 37,04 & 3,36 & 11,22 \\
2010 & 0,14 & 6,94 & 34,08 & 3,11 & 7,15 \\
2011 & 0,28 & 7,70 & 33,96 & 3,78 & 4,20 \\
2012 & 0,27 & 10,17 & 70,14 & 2,42 & 3,87 \\
2013 & 0,27 & 5,56 & 47,60 & 3,93 & 3,12 \\
2014 & 0,53 & 5,50 & 56,02 & 2,68 & 4,70 \\
2015 & 0,26 & 4,18 & 42,14 & 3,12 & 3,05 \\
2016 & 0,26 & 5,52 & 42,84 & 3,94 & 3,29 \\
\hline
\end{tabular}

Tabela 2: Taxa de incidência de cada agravo notificado por ano (por 10.000 habitantes), Barcarena, Pará, Brasil.

\begin{tabular}{lllllll}
\hline \multirow{2}{*}{ ANO } & \multicolumn{7}{l}{ DOENÇAS TROPICAIS NEGLIGENCIADAS } & & DENGUE \\
\cline { 2 - 7 } & LTA & LV & DC & TB & HANSENÍASE & 9,34 \\
2007 & 0,35 & 2,83 & 1,89 & 4,02 & 5,08 & 4,56 \\
2008 & 0,55 & 2,66 & 0,55 & 2,66 & 7,89 & 4,53 \\
2009 & 0,10 & 1,94 & 0,75 & 4,42 & 8,75 & 8,51 \\
2010 & 0,10 & 1,40 & 0,20 & 3,30 & 6,10 & 9,25 \\
2011 & 0,68 & 2,24 & 1,36 & 2,92 & 4,09 & 1,23 \\
2012 & 0,09 & 1,04 & 0,01 & 2,94 & 3,89 & 3,72 \\
2013 & 0,54 & 0,81 & 1,45 & 4,09 & 3,09 & 0,61 \\
2014 & 0,08 & 0,26 & 1,85 & 2,74 & 3,09 & 1,29 \\
2015 & 0,00 & 0,34 & 1,29 & 3,36 & 2,33 & 6,49 \\
2016 & 0,20 & 0,50 & 2,27 & 5,23 & 1,68 & \\
\hline
\end{tabular}

Dentre as doenças tropicais notificadas, entre 2007 e 2016, no município de Abaetetuba, a doença de Chagas foi a que apresentou maior registro (5.570 casos), seguido de leishmaniose visceral (916 casos) e hanseníase (764 casos). Em Barcarena, no mesmo período, dengue foi a que apresentou maior registro (595 casos), seguido de hanseníase e tuberculose (455 e 370, respectivamente).

Em ambos os municípios, leishmaniose tegumentar americana foi a que apresentou menor registro, sendo 37 casos, em Abaetetuba, e 28, em Barcarena. Na Tabela 3 estão descritos o número total de casos, a média dessa totalidade por ano e o coeficiente de incidência para cada doença, por município.

Tabela 3: Número total de casos, a média (por ano) e o coeficiente de incidência para cada doença, por Município.

\begin{tabular}{lllllll}
\hline \multirow{2}{*}{ DOENÇAS } & \multicolumn{2}{l}{ No TOTAL DE CASOS } & \multicolumn{2}{l}{ MÉDIA (TOTAL DE CASOS) } & \multicolumn{2}{l}{ MÉDIA (TAXA DE INCIDÊNCIA) } \\
\cline { 2 - 7 } LTA & M1* & M2* & M1 & M2 & M1 & M2 \\
LV & 37 & 28 & 3,7 & 2,8 & 0,25 & 0 \\
DC & 916 & 125 & 91,6 & 12,5 & 6,37 & 0,9 \\
TB & 5570 & 136 & 557 & 13,6 & 38,23 & 0,7 \\
HANSENÍ́ASE & 483 & 370 & 48,3 & 37 & 3,35 & 3,1 \\
DENGUE** & 764 & 455 & 76,4 & 45,5 & 5,36 & 4,2 \\
\hline
\end{tabular}

*M1 e M2: Abaetetuba e Barcarena, respectivamente.

** Dados não fornecidos pela Secretaria Municipal de Abaetetuba. 


\section{Crescimento populacional}

O crescimento populacional do município de Barcarena foi mais expressivo comparado ao de Abaetetuba neste estudo (Figura 3). A população de Barcarena, em 2007, era de 84.560 habitantes e, em 2016, estava em 118.537 habitantes, apresentando um crescimento de populacional de $40,18 \%$, ao passo que, a população de Abaetetuba, em 2007, era de 133.222 mil habitantes, e em 2016, estava em 151.934 mil habitantes, apresentando um crescimento populacional de $14,04 \%$.

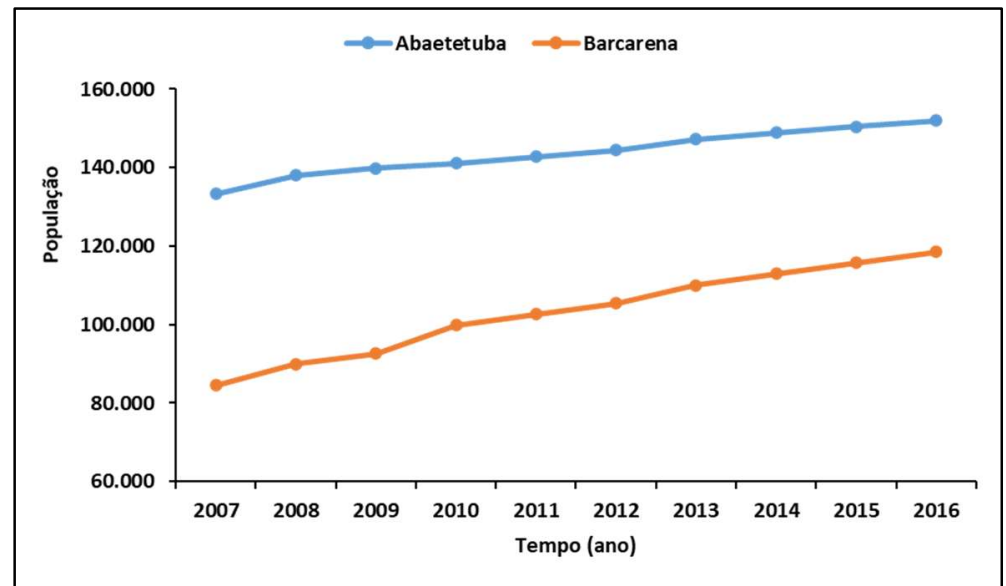

Figura 3: Crescimento Populacional nos municípios de Abaetetuba e Barcarena, Pará.

\section{Características socioeconômicas}

Conforme descrito nas Figuras 4 e 5, o Índice de Desenvolvimento Humano Municipal (IDHM) de Abaetetuba é 0,5, o que situa este município na faixa de IDHM Regular (IDHM entre 0,4 e 0,6), enquanto que, de Barcarena é 0,6, pertencendo a uma classificação de desenvolvimento moderado $(0,6$ a 0,8).

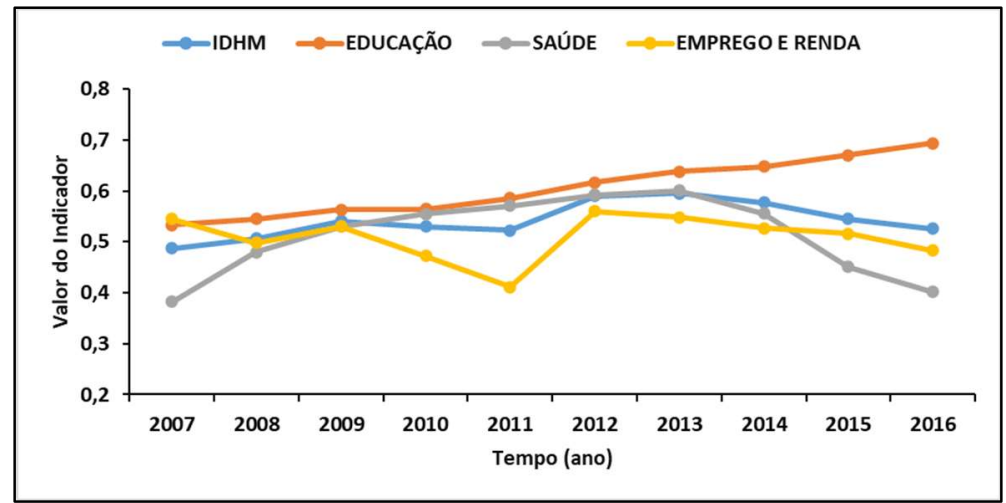

Figura 4: Índice de Desenvolvimento Humano Municipal geral e suas áreas do município de Abaetetuba, Pará.

O IDHM de Abaetetuba, no período estudado, foi de 0,5, o que situa este município na faixa de desenvolvimento regular (0,4 a 0,6). Na área da educação, seu índice foi de 0,5 (de 2007 a 2011) para 0,6 (de 2012 a 2016), passando de regular para moderado. Na área de emprego e renda, seu índice variava entre 0,4 e 0,5, mantendo-se na faixa de desenvolvimento regular. E na área da saúde, o índice oscilou bastante, pois em 2007 era 0,3 (baixo desenvolvimento), de 2008 a 2012 foi para 0,5 (desenvolvimento regular), 2013 chegou a 0,6 (desenvolvimento moderado), mas regrediu de 2014 a 2016, com índice igual a 0,4 (desenvolvimento regular). 


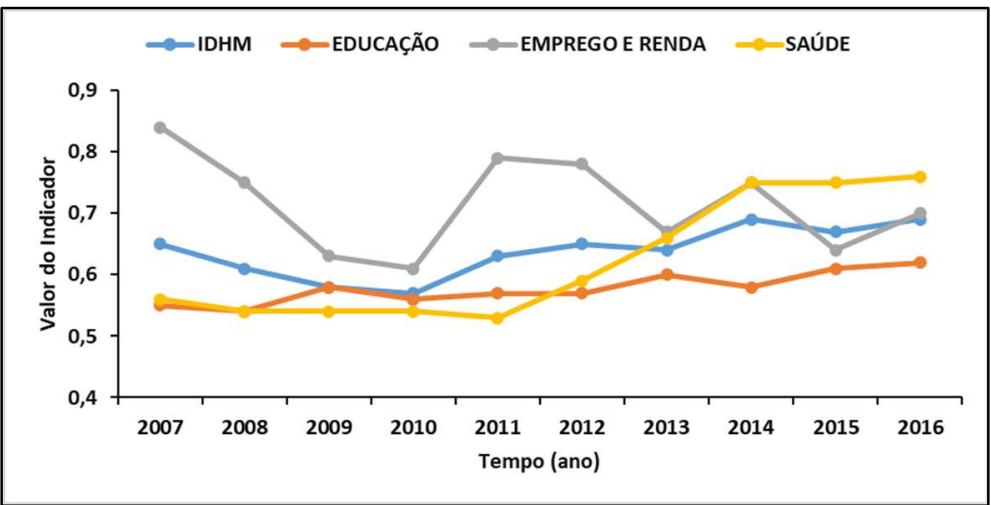

Figura 5: Índice de Desenvolvimento Humano Municipal geral e suas áreas do município de Barcarena, Pará.

Quanto ao IDHM de Barcarena, entre 2007 e 2016, foi de 0,6, o que o coloca na faixa de desenvolvimento moderado. Na área da educação, seu índice passou de 0,5 para 0,6, passando assim de desenvolvimento regular para desenvolvimento moderado. No setor de emprego e renda, o índice teve pequenas oscilações entre 0,6 e 0,7, mantendo-se sempre na faixa de desenvolvimento moderado. E, por fim, na área da saúde, o índice passou de 0,5 para 0,7, saindo da faixa de desenvolvimento regular para moderado.

\section{DISCUSSÃO}

As doenças tropicais são enfermidades infecciosas que se proliferam em condições climáticas quentes e úmidas, como boa parte do território brasileiro, e são chamadas de negligenciadas por atingirem, em sua maioria, uma população desprotegida social e economicamente (SOBRAL et al., 2018). Os municípios de Barcarena e Abaetetuba apresentaram dados relevantes quanto às DTNs e suas condições socioeconômicas, tendo como DTNs identificadas a leishmaniose tegumentar americana e visceral, doença de Chagas, tuberculose, hanseníase e dengue, sendo dengue presente apenas nos dados fornecidos pela Secretaria de Saúde do município de Barcarena.

A leishmaniose é uma doença negligenciada, endêmica em todas as regiões da OMS, que pode se manifestar de duas formas: tegumentar americana (LTA) ou cutânea e a visceral (LV) ou calazar (WHO, 2018a). Enquanto que na região europeia a Leishmaniose apresenta baixa carga global, no Brasil essa realidade é diferente, se apresentando como um dos cinco principais países com as maiores incidências deste agravo em todo o mundo (WHO, 2018a; TELES et al., 2019).

As taxas de incidência e seu coeficiente de incidência de LTA (Tabelas 1, 2 e 3) encontradas para os dois municípios foram baixas, ainda que em Abaetetuba esses dados tenham sido maiores comparados com Barcarena. No entanto, ambos não são condizentes com as taxas encontradas em outros trabalhos destes e de outros locais com dinâmicas semelhantes, podendo estar associada a diferentes relações entre uso e ocupação do solo (TELES et al., 2019; GONÇALVES et al., 2019; RODRIGUES et al., 2019).

A leishmaniose visceral apresentou alta incidência em Abaetetuba (média de 91,6 casos/ano), e no município de Barcarena foi considerada média (média de 12,5 casos/ano) de 2007 a 2016, sendo que a nível nacional, a média anual de casos de leishmaniose visceral foi de 3.565 casos (BRASIL, 2017a). O coeficiente 
de incidência foi de 6,37 e 0,9/10.000 habitantes, respectivamente, sendo bastante superior à média nacional, que foi de 0,19/10.000 habitantes, no período de 2003 a 2012 (BRASIL, 2017b).

O total de casos de leishmaniose visceral encontrados, no município de Abaetetuba, foi superior ao de outros achados, enquanto que de Barcarena corroboraram, pincipalmente no que se refere à redução dos índices desta doença neste município (TELES et al., 2014; SILVA et al., 2017; RODRIGUES et al., 2019). Tais resultados, tanto para LTA quanto para LV podem estar diretamente relacionados ao aumento do desmatamento e à carência de atendimento à saúde nessa região, fazendo com que os casos aumentem (SILVA et al., 2017; GONÇALVES et al., 2019).

A doença de Chagas é uma antropozoonose de elevada prevalência e expressiva morbimortalidade, principalmente na região Norte do país, mesmo com subnotificação bem expressiva, sobretudo no estado do Pará (BRASIL, 2015a). Ainda assim, alguns estudos mostram que o Pará é responsável por 95\% dos casos de doença de Chagas notificados no Brasil e 56 municípios apresentam risco de transmissão no Nordeste do Estado, com destaque para Belém, Abaetetuba, Barcarena, além da confirmação de que a via oral (consumo de açaí) tem sido a forma de contaminação mais frequente (BRASIL, 2015a; SOUSA JÚNIOR et al., 2017; VALENTE et al., 2018).

A média de casos desta doença foi de 557 casos/ano em Abaetetuba e de Barcarena foi de 13,6 casos/ano, enquanto que a nível nacional a média anual foi de 168 casos, entre 2008 e 2012 (BRASIL, 2017a). Em 2016, Barcarena apresentou casos recorrentes desta doença, não sendo possível quantificar esta recorrência (BRASIL, 2015a). Esta doença foi a que teve maior registro no município de Abaetetuba, não sendo possível informar se este alto índice foi resultado de uma contaminação por via oral ou vetorial.

De acordo com Sousa Júnior et al. (2017) foram identificados 83 casos de doença de Chagas no município de Barcarena, quantitativo este que corrobora com o presente estudo, se for levado em consideração o período dos mesmos (2007 a 2014). Ressalta-se, porém, que o presente estudo corresponde ao período de 2007 a 2016.

A tuberculose é uma doença infectocontagiosa de elevada magnitude e importância no mundo, causada pelo Bacilo de Koch (BK) ou Mycobacterium tuberculosis, que afeta principalmente os pulmões, com uma estimativa de, aproximadamente, um terço da população mundial infectada (COSTA, 2018; BRASIL, 2015b). O Brasil faz parte do grupo dos 22 países de alta carga de tuberculose no mundo, ocupando a 16a posição em número absoluto de casos (BRASIL, 2015b).

No país, no período de 2005 a 2014, foram diagnosticados, em média, 73 mil casos novos de tuberculose por ano, e em 2013, ocorreram mais de 4 mil óbitos (NEVES et al., 2018). No estado do Pará, em 2018, foram registrados quase 3.500 casos, com Belém sendo a segunda capital da região Norte com maior número de casos registrados (BRASIL, 2019a). No período compreendido entre 2007 e 2016 foram registrados 483 casos deste agravo em Abaetetuba, e 370 em Barcarena, com um coeficiente de incidência de 3,35 e 3,1/10.000 habitantes, respectivamente.

Até 2018 apenas oito estados brasileiros haviam implantado o Protocolo de Vigilância da Infecção Latente pelo Mycobacterium tuberculosis (ILTB), como medida criada em busca da redução da incidência e 
da mortalidade por tuberculose no país (BRASIL, 2019a). Medidas como essas são necessárias, pois nos dois últimos anos houve um aumento no coeficiente de incidência da tuberculose a nível nacional, e isso pode estar relacionado às mudanças no contexto social e econômico do país nos últimos anos (BRASIL, 2018 b).

Com relação à hanseníase, no município de Abaetetuba foi registrado um total de 764 casos entre 2007 e 2016, com uma média de 76,4 casos/ano e coeficiente de 5,36/10.000 habitantes. No município de Barcarena, a hanseníase foi a segunda doença com maior registro neste estudo, sendo 455 casos no total e, consequentemente, segunda maior com uma média de 45,5 casos/ano e um coeficiente de 4,2/10.000 habitantes, sendo considerada hiperendêmica nos dois municípios.

A OMS elaborou a Estratégia Global de Hanseníase 2016-2020 que deve ser adotada pela maioria dos países nos quais a hanseníase é endêmica e que serviu de base para a elaboração da Estratégia Nacional para o Enfrentamento da Hanseníase 2019-2022, cujo objetivo geral é contribuir para a redução da carga de hanseníase no Brasil (WHO, 2018b; BRASIL, 2019b). No território amazônico essas ações mitigadoras acabaram resultando em aumento dos registros da incidência de hanseníase, o que provavelmente deve estar relacionado ao maior investimento nas medidas de erradicação das doenças tropicais na Atenção Primária em Saúde, culminando com o aumento das notificações (TEIXEIRA et al., 2017).

Ainda assim, o estado do Pará ocupa o terceiro lugar entre os estados brasileiros com mais notificações de hanseníase no país, e por este motivo é considerado estado prioritário para o controle da mesma (SILVA et al., 2018; BRASIL, 2019b). Medidas de combate à erradicação da hanseníase são necessárias, visto que, enquanto a nível global, o número de novos casos detectados diminuiu modestamente, em 2017, o Brasil seguiu na contramão com 1.657 novos casos (WHO, 2018b).

Os dados sobre dengue foram disponibilizados apenas pela Secretaria Municipal de Barcarena, e foi a doença com maior registro, e, consequentemente, maior taxa de incidência, com 595 casos no total, uma média de 59,5 casos/ano e um coeficiente de incidência de 4,5/10.000. Interessante frisar que o coeficiente de incidência ao longo dos anos oscila bastante, como de 2007 que estava 9,34/10.000 habitantes, e no ano seguinte caiu para 4,56/10.000, sendo uma redução de quase $50 \%$ nesta taxa. Outro exemplo dessas oscilações se deu de 2015 para 2016, quando teve um aumento de 503\% no coeficiente de incidência de dengue neste município, passando de 1,29 para 6,49/10.000 habitantes.

Em 2016, foram registrados 1.487 .924 casos prováveis de dengue no país, com um coeficiente de 72,76 casos/10 mil habitantes, com destaque para o estado do Pará que, para efeito de comparação, em 2015 teve 7.505 casos e no ano seguinte teve 10.787 casos de dengue registrados (BRASIL, 2016). Até fevereiro deste ano foram registrados 54.777 casos prováveis de dengue no país, com um coeficiente de incidência de 2,63 casos/10 mil habitantes (BRASIL, 2019c).

A intensidade da transmissão da dengue é influenciada pela densidade populacional e fatores ecológicos (WHO, 2018c), o que pode ser justificado neste estudo visto que a população de Barcarena teve um aumento de mais de 40\% no período entre 2007 e 2016 (Figura 3). Apesar de a Secretaria Municipal de Saúde não ter fornecido dados sobre dengue no município de Abaetetuba, sabe-se que ela é uma doença frequente nesta localidade e que seu índice está diretamente ligado às baixas condições de saneamento 
básico local (RIBEIRO et al., 2015).

No que concerne ao crescimento populacional, notou-se que a população de Barcarena cresceu bastante neste mesmo período, no entanto, Abaetetuba manteve um número maior de habitantes, além de apresentar elevadas taxas das doenças tropicais negligenciadas em comparação com o município de Barcarena. Um fato que poderia justificar esses altos índices é o crescimento populacional desordenado, já que ele influencia diretamente neste processo (LIMA-CAMARA, 2016).

A crença no desenvolvimento baseado na industrialização, sob o comando das grandes potências, que promoveria então a melhoria da humanidade é um tema que marca os séculos XIX e XX (SOUZA, 2018). A exemplo tem-se o município de Barcarena que se destaca como pólo industrial de grande importância para o Estado do Pará, abrigando empreendimentos, principalmente da área minero-metalúrgica, atraindo um intenso fluxo migratório para a região pelas oportunidades de negócios e utilização de mão de obra barata no Município, favorecendo ocupações desordenadas do território e constantes transformações ambientais (TELES et al., 2014; ALVES et al., 2015)

Entretanto, com relação ao IDHM, não há muita diferença entre Barcarena e Abaetetuba, mostrando que a população de ambos os municípios se encontram em grande vulnerabilidade socioeconômica, e que há necessidade da implementação de programas que visem a melhoria na qualidade de vida de seus habitantes. Medeiros et al. (2016) já relataram que no município de Barcarena há problemas como a falta de serviços adequados de saneamento básico, por exemplo, causando assim, graves problemas ao meio ambiente e à população.

\section{CONCLUSÕES}

A elevada ocorrência de várias doenças tropicais negligenciadas nos dois municípios analisados e seus baixos índices de IDHM sugerem que há uma correlação entre eles, apesar de Barcarena ser um município que fez parte dos Grandes Projetos na Amazônia, através da implementação da Empresa Albrás/Alunorte. Em tese, este município deveria oferecer melhores condições de vida à sua população no que tange à saúde, saneamento básico e educação, por se tratar de um pólo industrial. No entanto, o que se vê são notificações de agravos que estão, comprovadamente em vários estudos científicos, correlacionados com as baixas condições socioeconômicas da sua população.

O município de Abaetetuba, por sua vez, aparenta carregar os ônus desse desenvolvimento no município vizinho, uma vez que, abriga boa parte da massa trabalhadora de Barcarena, sofrendo com inchaço populacional, população em condições de vulnerabilidade social e altos índices de casos de DTNs notificadas por sua Secretaria de Saúde, ainda que, suspeita-se de subnotificação para dengue.

Diante do exposto, propõe-se que as medidas para a erradicação das DTNs estejam acessíveis a todos os habitantes dos municípios em questão, intensificando suas ações, bem como ampliando os investimentos em educação e saneamento básico para que as iniquidades sociais sejam minimizadas. 


\section{REFERÊNCIAS}

ABAETETUBA. Plano Municipal de Saneamento do Município de Abaetetuba 2017. Diagnóstico dos serviços de saneamento. Abaetetuba: Secretaria Municipal de Meio Ambiente, 2017.

ALVES, R. J. M.; ROCHA, L. C. F.; PONTES, A. N.; COSTA, M. S. S.; CAMPOS, P. S. S.. Estudo socioeconômico de comunidades da área do polo industrial de Barcarena, Pará, Brasil. Enciclopédia Biosfera, v.11, n.21, 2015.

ARAÚJO, M. A. T.; BELO, P. S.. Grandes projetos minerários e comunidades tradicionais na Amazônia: impactos e perspectivas. Rev. Pol. Públ., v.13, n.2, p.265-77, 2009.

BOOTH, M.; CLEMENTS, A.. Neglected tropical disease control: the case for adaptive, location-specific solutions. Trends in Parasitology, v.34, n.4, 2018. DOI: https://doi.org/10.1016/j.pt.2018.02.001

BRANDÃO, V. V. P.; SILVA, J. M. P.; LIMA, J. B.; ANDRADE, A. S.. Indústria minerometalúrgica e a reorganização socioespacial no município de Barcarena/PA. In: Perspectivas e análises do espaço geográfico: Dinâmicas Urbano-Regionais e Ordenamento Territorial. Belém: UFPA, 2018. p.233-54.

BRASIL. Doença de Chagas aguda no Brasil: série histórica de 2000 a 2013. Brasília: Ministério da Saúde, 2015a.

BRASIL. Detectar, tratar e curar: desafios e estratégias brasileiras frente à tuberculose. Brasília: Ministério da Saúde, 2015b.

BRASIL. Guia de Vigilância em Saúde: volume 3. Brasília: Ministério da Saúde, 2017b.

BRASIL. Guia de Vigilância em Saúde: volume único. Brasília: Ministério da Saúde, 2017a.

BRASIL. Brasil Livre da Tuberculose: evolução dos cenários epidemiológicos e operacionais da doença. Brasília: Ministério da Saúde, 2019a.

BRASIL. Estratégia nacional para o enfrentamento da hanseníase: 2019 - 2022. Brasília: Ministério da Saúde, 2019b.

BRASIL. Monitoramento dos casos de Arboviroses urbanas transmitidas pelo Aedes (dengue, chikungunya e Zika) até a Semana Epidemiológica 5 de 2019. Brasília: Ministério da Saúde, 2019c.

BRASIL. Monitoramento dos casos de dengue, febre de chikungunya e febre pelo vírus Zika até a Semana Epidemiológica. Brasília: Ministério da Saúde, 2016.

BRASIL. Plano integrado de ações estratégicas de eliminação da hanseníase, filariose, esquistossomose e oncocercose como problema de saúde pública, tracoma como causa de cegueira e controle das geohelmintíases: plano de ação 2011-2015. Brasília: Ministério da Saúde, 2012.

BRASIL. Protocolo de vigilância da infecção latente pelo Mycobacterium tuberculosis no Brasil. Brasília: Ministério da Saúde, 2018b.

BRASIL. Situação epidemiológica e estratégias de prevenção, controle e eliminação das doenças tropicais negligenciadas no Brasil, 1995 a 2016. Brasília: Ministério da Saúde, 2018a.

BUCKEE, C. O.; TATEM, A. J.; METCALF, C. J. E.. Seasonal population movements and the surveillance and control of infectious diseases. Trends in Parasitology, v.33, n.1, 2017. DOI: $\underline{\text { https://doi.org/10.1016/i.pt.2016.10.006 }}$

CATETE, C.; GUIMARÃES, L.; SILVA, B.; SILVA, L.; DUARTE, C.; ALMEIDA, G.; SOARES, N.; LEÃO, K.; GUIMARÃES, R.. Geotecnologias aplicadas ao monitoramento de áreas degradadas e sua relação com casos de leishmaniose, Barcarena (PA), Brasil. In: SIMPÓSIO NACIONAL RECUPERAÇÃO DE ÁREAS DEGRADADAS, 11. Anais. Curitiba, Paraná: SOBRADE, 2017.

COSTA, F. A. C.. Neglected diseases and the Unified. Health System, ReonFacema, v.4, p.902-3, 2018.

ENGELMAN, D. L.; FULLER, C.; SOLOMON, A. W.; MCCARTHY, J. S.; HAY, R. J.; LAMMIE, P. J.; STEER, A. C.. Opportunities for integrated control of neglected tropical diseases that affect the skin. Trends in Parasitology, v.32, n.11, 2016. DOI: http://dx.doi.org/10.1016/j.pt.2016.08.005

FAPESPA. Diretoria de Estatística e de Tecnologia e Gestão da Informação. Estatísticas Municipais Paraenses. Belém: Barcarena Fundação Amazônia de Amparo a Estudos e Pesquisas, 2017.

FIRJAN. Federação das Indústrias do Estado do Rio de Janeiro. Índice FIRJAN de Desenvolvimento Municipal (IFDM) 2017. FIRJAN, 2017.

GONÇALVES, N. V.; MIRANDA, C. S. C.; COSTA, R. J. F.; GUEDES, J. A.; MATSUMURA, E. S. S.; COSTA, S. B. N.; NOGUCHI, S. K. T.; GUIMARÃES, L. H. R.; OLIVEIRA, R. A. C.; TAVARES, S. A. L.; CUNHA, V. R. M. P.; XAVIER, M.. Leishmaniose cutânea: distribuição espacial e fatores de risco ambiental no Estado do Pará, Amazônia Oriental Brasileira. The Journal of Infection in Developing Countries, v.13, p.939-944, 2019. DOI: http://dx.doi.org/10.3855/jidc.11573

HOFSTRAAT, K.; BRAKEL, W. H.. Social stigma towards neglected tropical diseases: a systematic review. International Health, v.8, n.1, p.i53-i70, 2016. DOI: http://dx.doi.org/10.1093/inthealth/ihv071

IBGE. Instituto Brasileiro de Geografia e Estatística. Estimativas da população residente no Brasil e Unidades da Federação com data de referência em 10 de julho de 2019. Rio de Janeiro: IBGE, 2019.

INÁCIO, M.; INVERNIZZI, N.. Nanotecnologias para doenças negligenciadas no Brasil: trajetórias de pesquisa, incentivos e perspectivas. Acta Scientiarum. Human and Social Sciences, v.41, 2019.

JACOBSON, J.; BUSH, S.. Neglected tropical diseases, neglected communities, and conflict: how do we leave no 
one behind. Trends in Parasitology, v.34, n.3, p.175-7, 2018.

LIMA-CAMARA, T. N.. Arboviroses emergentes e novos desafios para a saúde pública no Brasil. Rev. Saúde Pública, v.50, n.36, 2016. DOI: http://dx.doi.org/10.1590/S1518$\underline{8787.2016050006791}$

MEDEIROS, A. C.; LIMA, M. O.; GUIMARÃES, R.. Avaliação da qualidade da água de consumo por comunidades ribeirinhas em áreas de exposição a poluentes urbanos e industriais nos municípios de Abaetetuba e Barcarena no estado do Pará, Brasil. Revista Ciência e Saúde Coletiva, v.21, n.3, p.695708, 2016. DOI: http://dx.doi.org/10.1590/1413$\underline{81232015213.26572015}$

MENGITSU, B.; SHAFI, O.; KEBEDE, B.; KEBEDE, F.; WORKU, D. T.; HERERO, M.; FRENCH, M.; KEBEDE, B.; MACKENZIE, C.; MARTINDALE, S.; KEBEDE, Z.; HIRPA, T.; FRAWLEY, H.; CROWLEY, K.; O'NEIL, M.; MCPHERSON, S.. Ethiopia and its steps to mobilize resources to achieve 2020 elimination and control goals for neglected tropical diseases: Spider webs joined can tie a lion. Int Health, v.8, n.1, p.i34-i52, 2016. DOI: https://doi.org/10.1093/inthealth/ihw007

NEVES, D. C. O.; LOUREIRO, L. O.; PAIVA, N. P.; OHNISHI, M. D. O.; RIBEIRO, C. D. T.. Análise do Programa de Controle da Tuberculose no estado do Pará, Brasil, de 2005 a 2014. Rev. Pan-Amaz. Saúde, v.9, n.4, p.47-56, 2018.

QUARESMA, M.; SOMBRA, D.; LEITE, A.; CASTRO, C.. Periodização econômica de Abaetetuba (PA) a partir de sua configuração espacial. Revista PerCursos, v.16, n.32, p.14368, 2015.

RIBEIRO, É. R. F.; ALENCAR, I. C.. Análise de risco da ocupação da Chicolândia em Abaetetuba (PA): uma proposta de gestão ambiental sustentável. GEOSABERES: Revista de Estudos Geoeducacionais, v.6, n.3, p.110-21, 2015.

RODRIGUES, M. G. A.; SOUSA, J. D. B.; DIAS, A. L. B.; MONTEIRO, W. M.; SAMPAIO, V. S.. The role of deforestation on American cutaneous leishmaniasis incidence: spatial-temporal distribution, environmental and socioeconomic factors associated in the Brazilian Amazon. Tropical Medicine and International Health, v.24, n.3, p.348-55, 2019. DOI: http://dx.doi.org/10.1111/tmi.13196

SILVA, B. C. S.; CATETE, C. P.; GUIMARÃES, L. H. R.; SILVA, L. C. T.; GUIMARÃES, R. J. P. S.. Análise espacial da leishmaniose visceral no município de Abaetetuba/PA, no período de 2007 a 2015. In: CONGRESSO DA ASSOCIAÇÃO BRASILEIRA DE ENGENHARIA SANITÁRIA E AMBIENTAL, 29. Anais. São Paulo: FENASAN, 2017.

SILVA, J. S. R.; PALMEIRA, I. P. P.; SÁ, A. M. M.; NOGUEIRA, L. M. V.; FERREIRA, A. M. R.. Fatores sociodemográficos associados ao grau de incapacidade física na hanseníase. Rev. Cuid., v.9, n.3, p.1-11, 2018. DOI: http://dx.doi.org/10.15649/cuidarte.v9i3.548

SOBRAL, N. V.; MIRANDA, Z. D., MASCARENHAS, F. S.. Estratégia para a recuperação de informação científica sobre as doenças tropicais negligenciadas: análise comparativa da Scopus, Pubmed e Web of Science. Revista Cubana de Información en Ciências de la Salud, v.29, n.1, p.35-53, 2018.

SOUSA JÚNIOR, A. S.; PALÁCIOS, V. R. C. M.; MIRANDA, C. S.; COSTA, R. J. F.; CATETE, C. P.; TELES, E. J. C.; PEREIRA, A. L. R. R.; GONÇALVES, N. V.. Análise espaço-temporal da doença de Chagas e seus fatores de risco ambientais e demográficos no município de Barcarena, Pará, Brasil. Rev. Bras.

Epidemiol, v.20, n.4, p.742-755. 2017. DOI:

http://dx.doi.org/10.1590/1980-5497201700040015

SOUZA, C. M.. A região dos desejos e das aventuras: diálogos sobre grandes projetos de integração e desenvolvimento na Amazônia nos séculos XIX e XX. Saúde e Meio Ambiente, v.31, n.48, 2018. DOI:

http://dx.doi.org/10.22562/2018.48.02

TEIXEIRA, R. L.; NUNES, L. M.; SANTOS, B. E. F.; ARAÚJO, M. H. M.; BUENO, A. C.; COUTINHO, T. S.. Perfil epidemiológico dos pacientes de 0 a 15 anos de idade com hanseníase. Rev. Med. Saúde, Brasília, v.6, n.3, p.291-302, 2017.

TELES, E. J. C.; SOARES, D. C.; GASPARETTO, D.; VEIGA, N.; FONTELLES, M. J. P.; GARCEZ, L. M.. Leishmaniose visceral no Município minerário de Barcarena, Estado do Pará, Brasil: evolução de 2004 a 2008 e bases para a vigilância epidemiológica. Rev. Pan-Amaz. Saúde, v.5, n.2, p.21-7, 2014.

TELES, G. C.; FONSECA, F. R.; GONÇALVES, M. J. F.. Leishmaniose Tegumentar Americana na Amazônia Brasileira de 2010 a 2014. São Paulo: Rev. Inst. Med. Trop., v.61, 2019. DOI: http://dx.doi.org/10.1590/s1678-9946201961022

VALENTE, V. C.; PINTO, A. Y. N.; RAMOS, F. L.; MATOS, H. J.; PEREIRA, L. C. S.; MONTEIRO, É. A.. Doença de Chagas aguda no Estado do Pará: casuística do Instituto Evandro Chagas no ano de 2017. In: CONGRESSO DA SOCIEDADE BRASILEIRA DE MEDICINA TROPICAL, 54. Anais. Recife: SBMT, 2018.

WHO. World Health Organization. Água, saneamiento e higiene para acelerar y mantener el progresso: Estrategia mundial de 2015 a 2020. Geneva: WHO, 2015.

WHO. World Health Organization. Integrating neglected tropical diseases into global health and development: fourth WHO report on neglected tropical diseases. Geneva: WHO, 2017.

WHO. World Health Organization. Surveillance of leishmaniasis in the WHO European Region. Geneva: WHO 2018a.

WHO. World Health Organization. Dengue vaccine: WHO position paper. Weekly Epidemiological Record. no 36. Geneva: WHO, 2018c.

WHO. World Health Organization. Global leprosy update, 2017: reducing the disease burden due to leprosy. Weekly Epidemiological. Geneva: WHO, 2018b.

A CBPC - Companhia Brasileira de Produção Científica (CNPJ: 11.221.422/0001-03) detém os direitos materiais desta publicação. Os direitos referem-se à publicação do trabalho em qualquer parte do mundo, incluindo os direitos às renovações, expansões e disseminações da contribuição, bem como outros direitos subsidiários. Todos os trabalhos publicados eletronicamente poderão posteriormente ser publicados em coletâneas impressas sob coordenação da Sustenere Publishing, da Companhia Brasileira de Produção Científica e seus parceiros autorizados. Os (as) autores (as) preservam os direitos autorais, mas não têm permissão para a publicação da contribuição em outro meio, impresso ou digital, em português ou em tradução. 